\title{
Molecular Mechanisms Involved in Inflammation and Insulin Resistance in Chronic Diseases and Possible Interventions
}

\author{
Renata Gorjão, ${ }^{1}$ Hilton Kenji Takahashi, ${ }^{2}$ Ji An Pan, ${ }^{3}$ and Sandro Massao Hirabara ${ }^{1,4}$ \\ ${ }^{1}$ Post-Graduate Program in Human Movement Sciences, Institute of Physical Activity Sciences and Sports, \\ Cruzeiro do Sul University, 01506-000 São Paulo, SP, Brazil \\ ${ }^{2}$ Institut de Recherche Expérimentale et Clinique, Université Catholique de Louvain, 1200 Brussels, Belgium \\ ${ }^{3}$ Department of Molecular Genetics and Microbiology, Stony Brook University, 216A Life Sciences Building, \\ Stony Brook, NY 11794-5222, USA \\ ${ }^{4}$ Post-Graduate Program in Biotechnology, Department of Physiology and Biophysics, Institute of Biomedical Sciences, \\ University of São Paulo, Avenida Professor Lineu Prestes, 1524, Butanta, SP, Brazil
}

Correspondence should be addressed to Sandro Massao Hirabara, sandromh@yahoo.com.br

Received 16 September 2012; Accepted 16 September 2012

Copyright (C) 2012 Renata Gorjão et al. This is an open access article distributed under the Creative Commons Attribution License, which permits unrestricted use, distribution, and reproduction in any medium, provided the original work is properly cited.

Inflammation and insulin resistance are present in several chronic diseases, including obesity, type 2 diabetes mellitus, metabolic syndrome, cancer, and cardiovascular diseases. Recent studies showed a close relationship between these two conditions, although the precise mechanisms are not completely understood. This special issue aimed to join original research and review articles related to the involvement of inflammation and/or insulin resistance with the development of chronic diseases as well as the interaction between these two factors and possible interventions based on important molecular targets. This issue was interested in articles that explore molecular aspects of inflammatory pathways, insulin resistance, and the crosstalk between them, in humans and in cell and animal models. Moreover, we accepted studies that explored interventions based on molecular targets for preventing or treating correlated disorders and advances for a better characterization and understanding of the mechanisms and mediators involved with the different inflammatory and insulin resistance conditions, addressing biotechnological studies for the development of new potential therapies and interventions. The potential topics for this special issue included (a) molecular basis of inflammation and insulin resistance; (b) crosstalk between inflammatory pathways and insulin signaling; (c) cell and animal models to test and understand the role of inflammation and insulin resistance as well as the interaction of these two factors on the development of chronic diseases; (d) interaction of inflammation and/or insulin resistance in human pathology conditions; (e) factors leading to inflammation and/or insulin resistance in models of chronic diseases; (f) identification of new biomarkers of chronic inflammation and insulin resistance in different models; (g) identification of new molecular targets for reducing inflammation and insulin resistance in chronic disease models; (h) new interventions for preventing or reducing insulin resistance and inflammation based on molecular targets in signaling pathways.

In the review article from S. Hirabara et al., the authors discussed the recent findings concerning insulin resistance and inflammation, the relationship between these two factors, possible mechanisms involved, and potential interventions for the disorders related. Authors pointed the advances in the field and new biotechnological tools and methodologies that have aided to understand these processes. Discovery and identification of new biomarkers involved with the development of chronic diseases characterized by increased inflammation and insulin resistance, as well as their relevance in the comprehension of interaction of these processes, were also discussed and will allow the study of treatment or prevention for related disorders.

L. Masi et al. characterized the effects of sunflower oil supplementation on insulin resistance and inflammation in mice submitted to high-fat diet (HFD). Briefly, the authors observed that the sunflower oil supplementation induces proinflammatory responses in macrophages and insulin-sensitive peripheral tissues, as well as insulin resistance. These responses were observed in control mice 
and pronounced in mice submitted to HFD. Interestingly, although sunflower oil supplementation was able to improve dislipidemia in mice fed with HFD, it increased inflammatory condition and insulin resistance state induced by the diet.

P. Li et al. studied gene variants of mitofusin-2 (MFN-2) in subjects with type 2 diabetes mellitus. This gene is involved in the mitochondrial fusion, regulating the morphology and distribution of this organelle, especially in cells and tissues with high demand of energy, including skeletal muscle and heart. Authors found a relationship between MFN-2 gene polymorphisms and type 2 diabetes mellitus, as well as between some MFN-2 gene variants and two other gene polymorphisms: peroxisome proliferator-activated receptor $\gamma$ coactivator- $1 \alpha$ (PGC- $1 \alpha$ ) and estrogen-related receptor- $\alpha$ (ESRRA) genes, which are important transcriptional factors that also regulate mitochondrial biogenesis and oxidative capacity. These findings point MFN-2 gene as a new potential target for further studies aiming at preventive or therapeutic interventions.

In the study of G. Rodrigues et al., authors investigated the effects of the treatment with aqueous extract from Croton cajucara plant on hepatic oxidative stress in streptozotocininduced diabetic rats. Croton cajucara is a Brazilian plant rich in several compounds, including flavonoids, coumarins, and alkaloids, that has been suggested to have important antioxidant effects, being used in various diseases, including diabetes mellitus, hypercholesterolemia, diarrhea, malaria, fever, gastrointestinal, renal, and hepatic disorders. The results found in this study suggest that the treatment with Croton cajucara aqueous extract is effective in decreasing hepatic oxidative stress and cell damage in streptozotocininduced diabetic rats, indicating for the potential therapeutic use of this plant in related diseases.

M.-Liao et al. reviewed the role of insulin resistance in the development of chronic kidney disease and the potential mechanisms involved. They discuss how different factors, including chronic inflammation, cellular and endoplasmic reticulum oxidative stress, decreased serum erythropoietin, elevated plasma adipokine and fetuin- $\mathrm{A}$, and vitamin $\mathrm{D}$ deficiency, can interfere in insulin sensitivity, contributing to the insulin resistance establishment and consequently to the development of chronic kidney disease.

C.-Sung et al. discussed the role of vitamin D on insulin resistance in several pathological conditions, including diabetes mellitus, hypertension, and cardiovascular diseases. Mechanisms for vitamin D deficiency associated with inherited gene polymorphisms are also presented, and its relevance in the immunoregulatory function and insulin resistance development is addressed. Although important advances in the field have been reached, the underlying mechanisms involved in these processes require further investigation.

G. Zhang et al., studying white rabbits, modified a protocol for the development of a new model of human atherosclerosis and vulnerable plaque, allowing a novel approach for related studies. Several markers of the disease were used in order to validate the model, including structural alterations, triacylglycerol and LDL-cholesterol serum levels, inflammatory parameters, and oxidative stress. This pattern of atherosclerosis for rabbits is very close to that in humans with several advantages when compared to other animal models, showing potential applicability and clinical relevance.

C. Leandro et al. focused on gestational insulin resistance and low insulin secretion induced by undernutrition during pregnancy. They found that low-protein diet during gestation impairs the glucose-stimulated insulin secretion and glucose tolerance. In contrast, a program of pregestational and gestational moderate physical training improved the glucose-stimulated insulin secretion and partially prevented the effects of perinatal undernutrited rats. Thus, wellcontrolled programs of moderate physical training, which have well-characterized systemic anti-inflammatory effects, starting at early pregnancy, are a potential tool for preventing insulin resistance and gestational diabetes mellitus during late pregnancy, as well as for increasing insulin secretion stimulated by glucose and avoiding deleterious effects on the offspring.

A. Alfadda and R. Sallam discussed in their review article the biological functions of reactive oxygen species (ROS) in several physiological and pathological processes. The importance of physiological ROS production on normal vascular diameter regulation, cellular oxygen sensing, immune system function, skeletal muscle physiology, cellular signaling pathways, and gene expression control is presented. On the other hand, the effects of excessive ROS production on the development of diseases, including cancer, diabetes mellitus, obesity, chronic inflammation, cardiovascular diseases, and metabolic syndrome are also discussed. This review provides recent findings and advances in this area for understanding the functions of ROS on different targets during various conditions in health and disease.

\section{Acknowledgments}

The editors of the special issue "molecular mechanisms involved in inflammation and insulin resistance in chronic diseases and possible interventions" on Journal of Biomedicine and Biotechnology (JBB) thank the JBB Editorial Office and all authors for providing important contributions in this issue, helping to understand the molecular mechanisms involved in these conditions and addressing further research in this field. The contributions in this issue discuss novel insights, potential treatment interventions, and new molecular targets involved in the establishment of insulin resistance and inflammatory state as well as the interaction between these two factors.

Renata Gorjão Hilton Kenji Takahashi Ji An Pan Sandro Massao Hirabara 

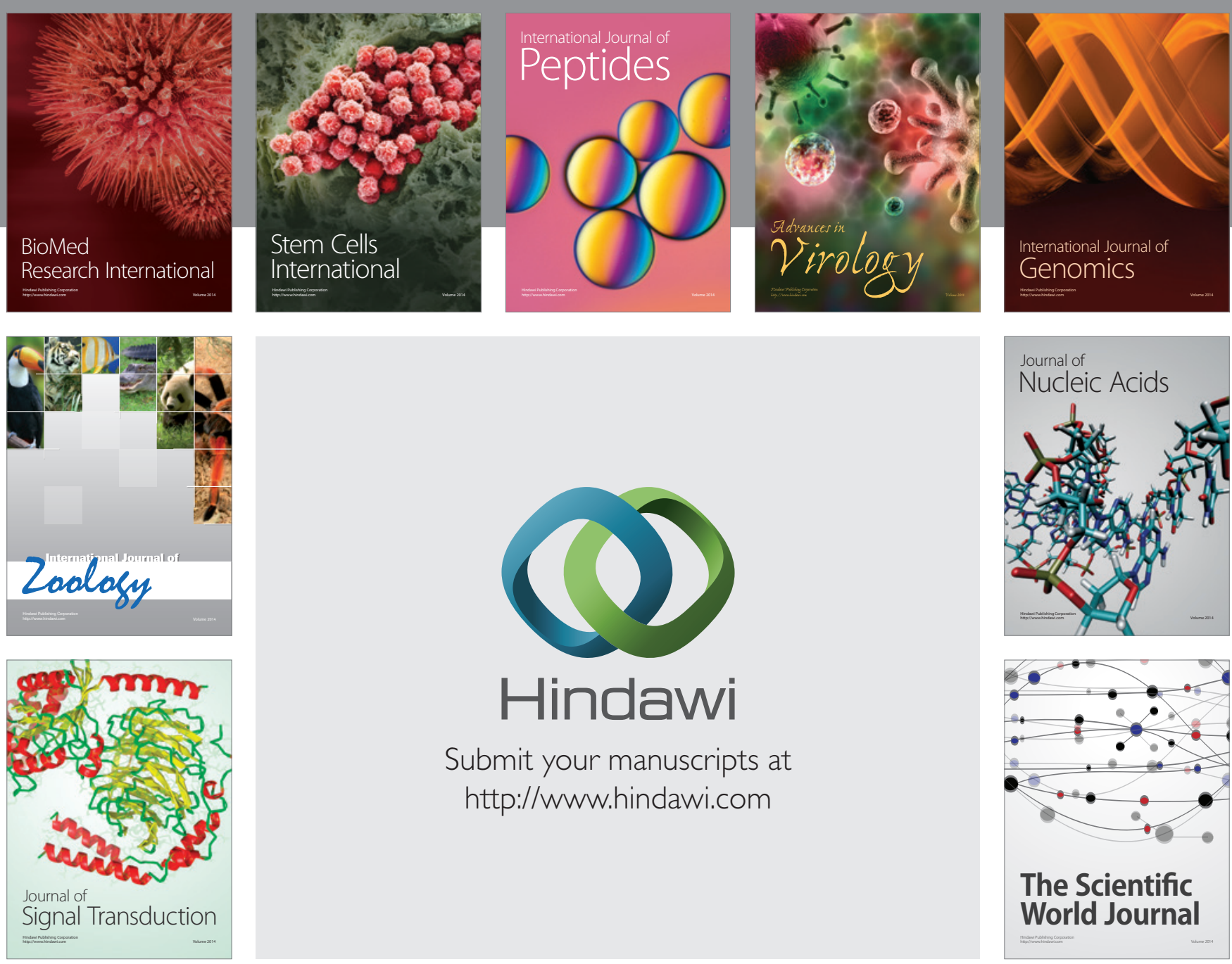

Submit your manuscripts at

http://www.hindawi.com
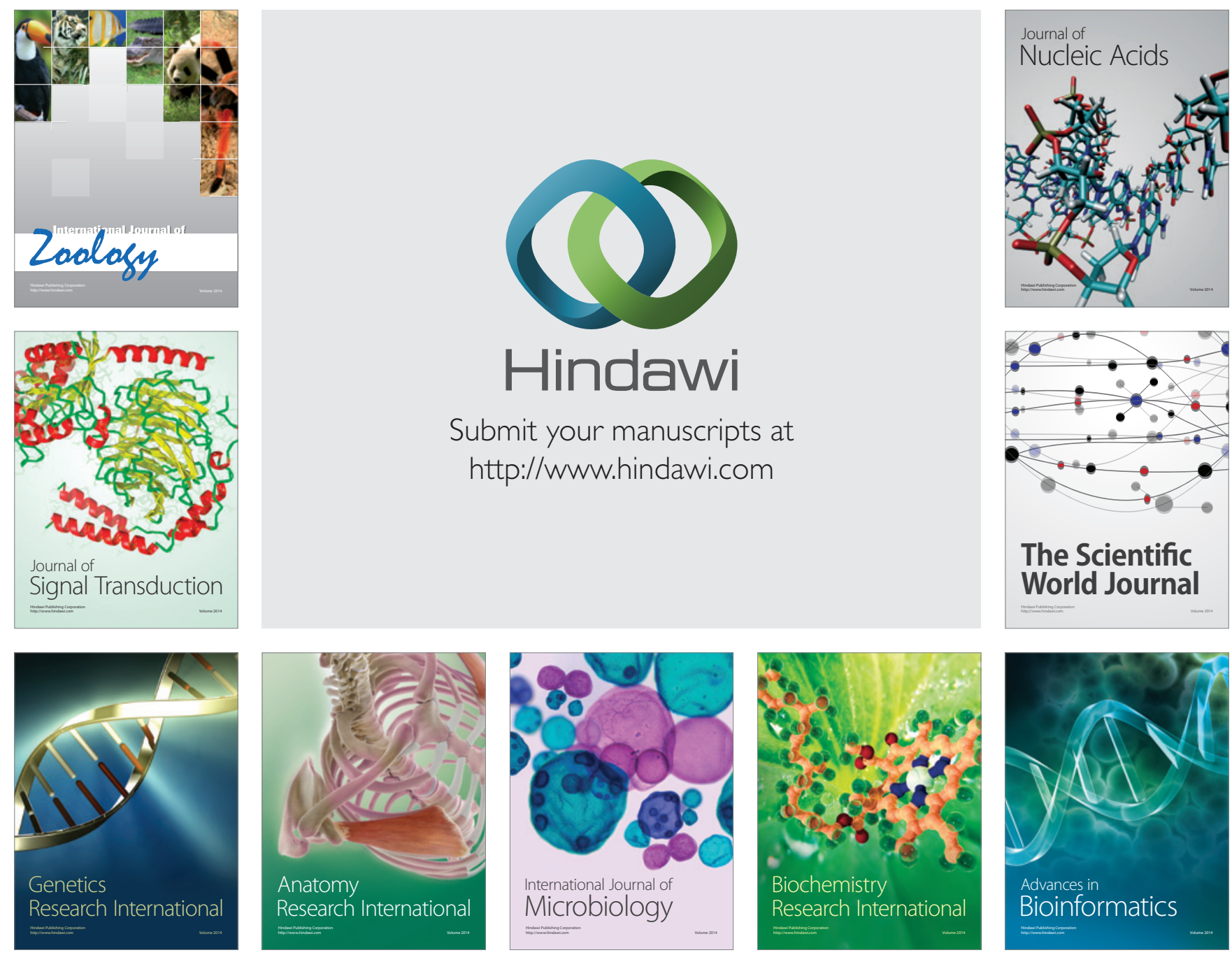

The Scientific World Journal
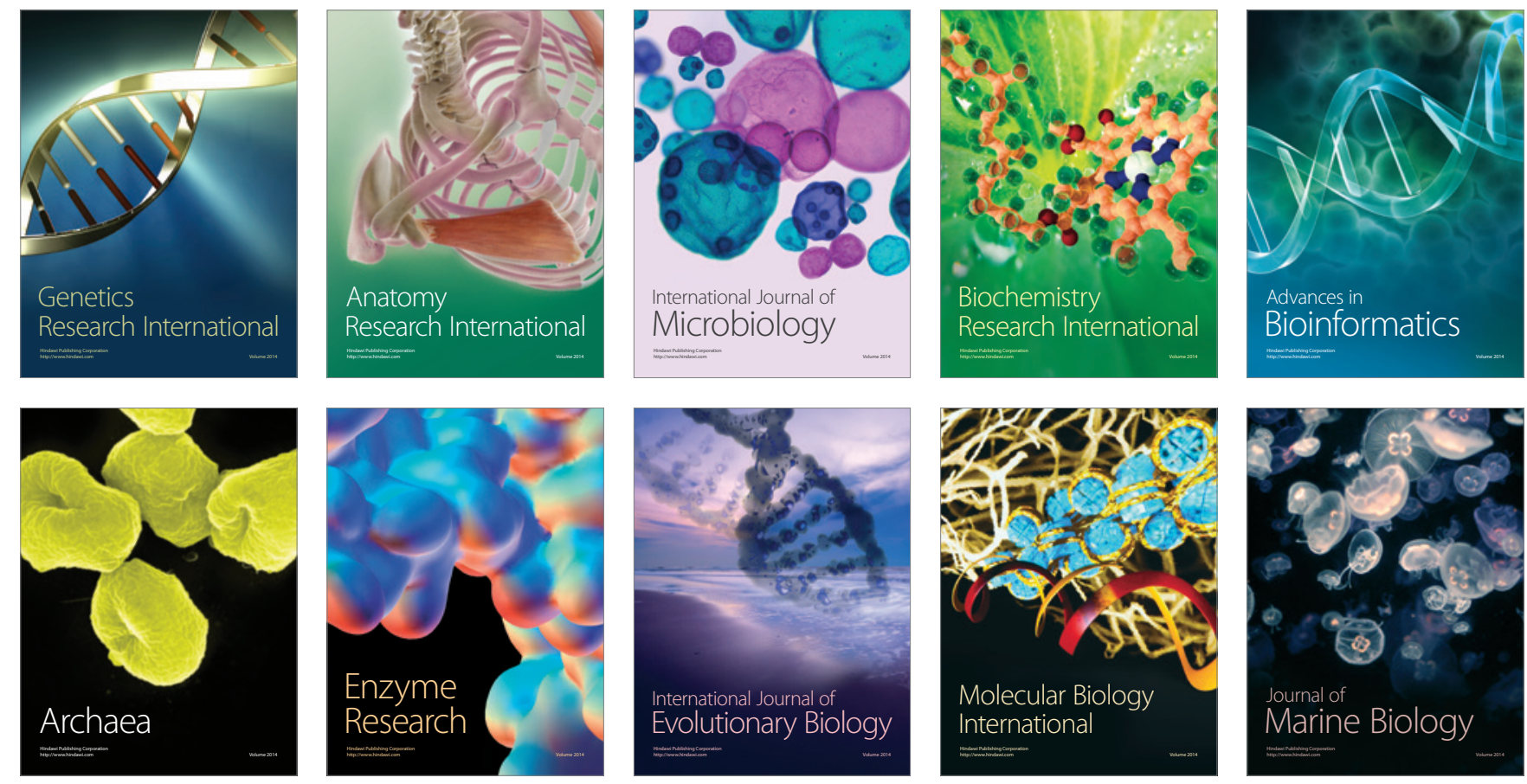